\title{
Monitoring of a flat plate solar thermal field supplying process heat
}

\author{
Marco Cozzini ${ }^{1, *}$, Roberto Fedrizzi ${ }^{1}$, Mauro Pipiciello ${ }^{1}$, Robert Söll ${ }^{2}$, Ilyes Ben Hassine ${ }^{3}$, and Dirk Pietruschka ${ }^{3}$ \\ ${ }^{1}$ EURAC Research, Institute for Renewable Energy, Viale Druso 1, 39100 Bolzano, Italy \\ 2 S.O.L.I.D. Solarinstallationen und Design GmbH, Puchstrasse 85, 8020 Graz, Austria \\ ${ }^{3}$ Research Center on Sustainable Energy Technology, Schellingstraße 24, 70174 Stuttgart, Germany
}

\begin{abstract}
The article reports the performance data of a flat plate collector field installed in Austria and supplying process heat to a meat factory, up to a temperature of about $95^{\circ} \mathrm{C}$. The presented data span an entire year, thereby including seasonal effects and allowing for a full characterization of the system performances. Sensor uncertainty is also discussed in detail. Finally, a bin method analysis of the field efficiency is provided. To this purpose, different operating conditions are concisely represented by the so-called reduced temperature, typically used in solar collector applications.
\end{abstract}

\section{Introduction}

Solar process heat is an application with an interesting potential, due to the large number of manufacturing technologies requiring heat in the low-medium temperature range [1,2]. According to statistics, about $27 \%$ of the European total energy demand is devoted to industrial heat, with a share of $30 \%$ in the low temperature range (i.e., below $100{ }^{\circ} \mathrm{C}$ ) and a share of $27 \%$ in the medium temperature range (i.e., between 100 and $400^{\circ} \mathrm{C}$ ). This kind of data was collected in specific European projects (see, for example, Ecoheatcool [3]) and was confirmed in Tasks (e.g., Task 33 and 45) of the Solar Heating and Cooling (SHC) programme of the International Energy Agency (IEA).

In the European FP7 project InSun, different demonstration activities were undertaken to investigate the reliability and efficiency of various collector technologies in different climatic regions: flat plate collectors, designed to obtain low temperatures (up to $100^{\circ} \mathrm{C}$ ) and suitable also for moderate northern Europe climates, and concentrating collectors (parabolic trough collectors and linear Fresnel collectors), designed to yield medium temperatures $\left(160-250^{\circ} \mathrm{C}\right)$ in the presence of proper radiation conditions, as in southern Europe climates. Here, the case of flat plate collectors is presented. The considered field - with an overall collector aperture area $A_{\mathrm{a}}$ of $990 \mathrm{~m}^{2}$ and a peak thermal power of about $600 \mathrm{~kW}$ - is installed in Austria, at Fleischwaren Berger Gmbh, a company producing meat and sausages [4].

The monitoring of a real application is of course of high interest for the solar community. Indeed, though standard tests according to EN 12975:2006 [5] (see also the more recent EN ISO 9806:2013 [6]) partially take into account

\footnotetext{
* e-mail: marco.cozzini@eurac.edu
}

transient effects with the so-called quasi-dynamic procedure, the behaviour of collectors in a real system can be affected by additional non-negligible phenomena [7-9], as dust and ageing.

\section{System layout}

The layout of the system is shown in Figure 1. The solar field consists of seven different arrays connected in parallel. Each array has a different number of flat plate collectors connected in series. In Figure 1, the gross area of each collector array is also reported. Flow rate within each array was balanced during the commissioning phase according to the corresponding area, in order to get a homogeneous outlet temperature.

The integration of the solar heat in the process is achieved with an intermediate heat-exchanger (HXs in Fig. 1), connected to the solar field on one side and to a large storage tank feeding the process lines on the other side. Depending on the temperature level, the solar heat output from the heat exchanger can be supplied either in the middle or at the upper part of the storage tank. Tank temperatures can vary along the day: for example the top temperature ranges from 55 to $95^{\circ} \mathrm{C}$ during a typical summer day, with an average of about $75^{\circ} \mathrm{C}$. The tank then supplies heat to two different processes:

- hot water production. Fresh water is first pre-heated with heat recovery (from chillers) up to $30-40{ }^{\circ} \mathrm{C}$. The solar contribution further rises temperature up to about $55^{\circ} \mathrm{C}$;

- feed water for steam production. Fresh water is preheated with heat recovery up to $30-40{ }^{\circ} \mathrm{C}$. The solar contribution then rises this temperature up to about $95^{\circ} \mathrm{C}$. Solar energy is hence applied to make up-water, rather than directly to feed water. Finally, feed water is obtained adding condensate. 


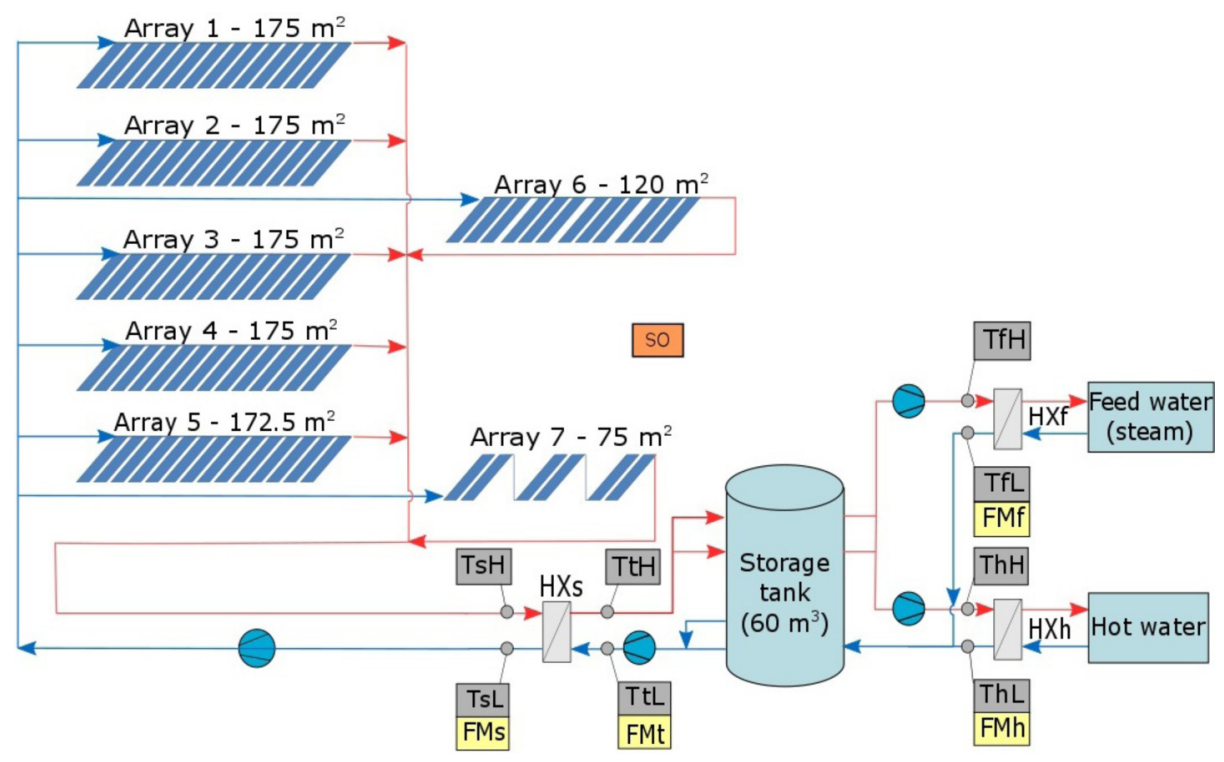

Fig. 1. Layout of the integration system. Temperature sensors are denoted by TxX $(X=H$, L for higher and lower temperature respectively; $\mathrm{x}=\mathrm{s}$, t, f, h for solar, tank, feed water and hot water circuit, respectively), flow meters by FMx, heat exchangers by HMx. Global irradiance on the tilted plane of the collectors is measured through a pyranometer, here denoted by SO.

\section{Monitoring results}

The positioning of the temperature and flow rate sensors considered in this paper is shown in Figure 1. In addition, a pyranometer measuring the global solar irradiance on the collector plane is present. The ambient temperature is instead obtained from a nearby weather station. For the present analysis, data have been averaged on a 10-min basis.

Below, the measurement uncertainties are discussed, describing the uncertainty propagation from the different sensors. While when assessing the efficiency of the entire system other significant uncertainty sources should be considered (e.g., cleaning of the collectors), it is useful to consider separately the uncertainty for direct measurements. This is especially interesting for industrial applications, where often economic reasons prevent the use of highly accurate sensors. On the other side, the statistical uncertainty associated to the field efficiency under different conditions is directly included in the bin method analysis reported later on in the article.

\subsection{Flow rate sensors}

These sensors are either of turbine type (solar field main pipe) or of ultrasonic type (remaining circuits). They hence provide the volumetric flow rate $Q_{\mathrm{v}}$. Their relative uncertainty $\sigma Q_{\mathrm{v}} / Q_{\mathrm{v}}$ is $<1.5 \%$ for the solar circuit and $<2 \%$ for the other circuits. The operating conditions for flow rate are typically very stable.

\subsection{Temperature sensors}

These are Pt1000 1/3 DIN sensors, with an uncertainty $\sigma_{T}=(1 / 3) \cdot(0.3+0.005|T|){ }^{\circ} \mathrm{C}$, where $T$ is the temperature in Celsius degrees. To calculate the thermal power, the inlet-outlet temperature difference $\Delta T=T_{\text {out }}-T_{\text {in }}$ is needed. Assuming uncorrelated sensors measuring similar temperatures, the uncertainty is about $\sigma_{\Delta T}=\sigma_{T} \sqrt{ } 2$. For the main solar circuit, $\Delta T$ typically lies in the $20-25 \mathrm{~K}$ range, so that $\sigma_{\Delta T} / \Delta T<2 \%$; however, operating conditions can vary along the day. In the other circuits, $\Delta T$ is usually higher and the relative uncertainty consequently lower.

\subsection{Thermal power and energy}

Thermal power is calculated as $P_{\text {th }}=Q_{\mathrm{v}} \rho(<T>) C_{\mathrm{p}}(<T>)$ $\Delta T$, where $\rho$ and $C_{\mathrm{p}}$ are the fluid density and specific heat respectively, while $\langle T\rangle=\left(T_{\text {in }}+T_{\text {out }}\right) / 2$ is the inletoutlet average temperature. The specific heat has a nonnegligible dependence on temperature, especially in the case of the solar circuit, where the thermal fluid is a mixture of water and propylene glycol (35\% vol.). In our calculations, temperature-dependent density and specific heat values were used, with a negligible contribution to uncertainty. Thereby, $\left.\quad \sigma_{P \text { th }} / P_{\text {th }}=\sqrt{ }\left(\sigma Q_{\mathrm{v}} / Q_{\mathrm{v}}\right)^{2}+\left(\sigma_{\Delta T} / \Delta T\right)^{2}\right]$. As seen above, the two contributing uncertainties are similar and the relative uncertainty for power is typically $<3 \%$. Thermal energy is calculated as $E_{\mathrm{th}}=\Delta t \Sigma_{i} P_{\mathrm{th}, i}$, where $\Delta t$ is the sampling time. It is expected that consecutive power measurements are correlated to some extent, as it can be deduced from the error model expected for the flow rate sensors. In order to estimate the energy uncertainty, we then adopt the most conservative choice: we assume fully correlated power measurements and thereby use a linear uncertainty propagation [10]. This yields $\sigma_{E \text { th }}=\Sigma_{i} \sigma_{P \text { th }, i}$ and hence $\sigma_{E \mathrm{th}} / E_{\mathrm{th}}=\left\langle\sigma_{P \mathrm{th}, i}\right\rangle /\left\langle P_{\mathrm{th}, i}\right\rangle$, where angle brackets represent the average on the considered number of power measurements. The final result for the relative uncertainty of energy is close to what reported above for power, i.e., $3 \%$. 


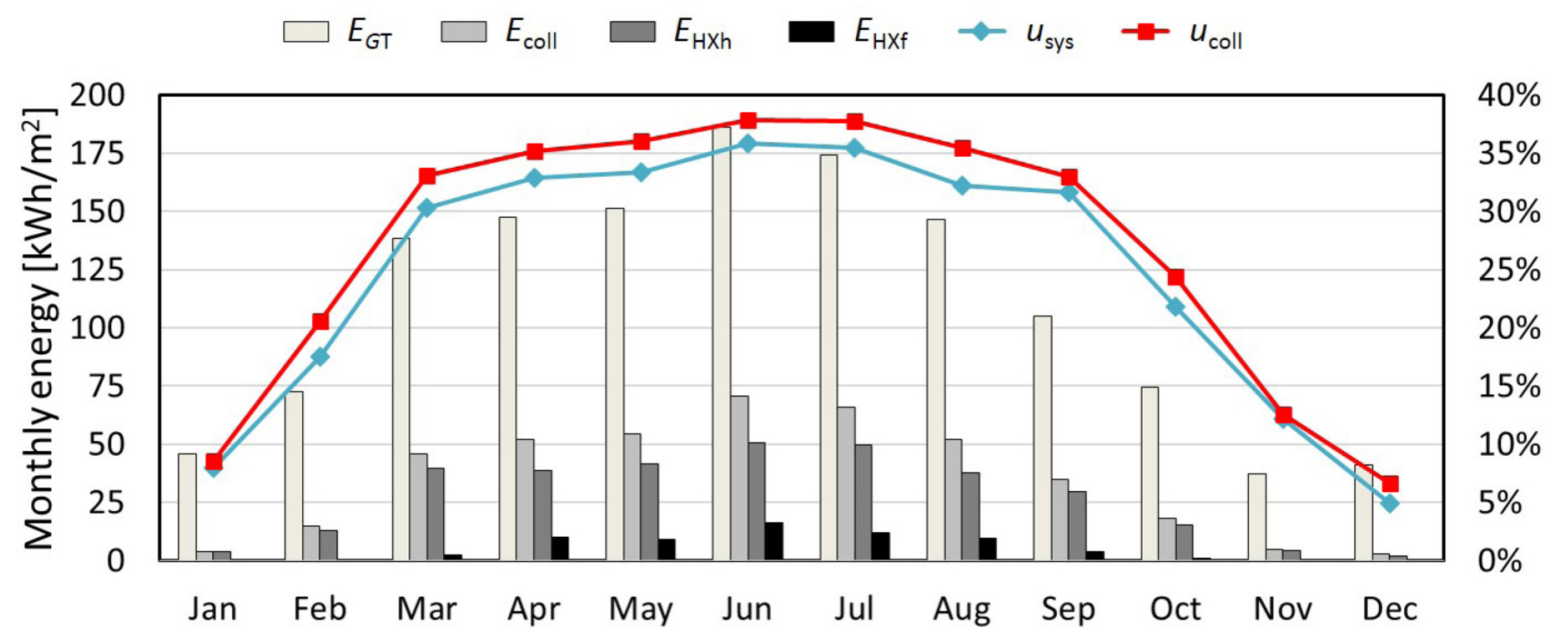

Fig. 2. Specific energy output and utilization ratio by month for 2014 .

\subsection{Solar irradiance and solar energy}

The global solar irradiance on the tilted $\left(40^{\circ}\right)$ collector plane, $G_{\mathrm{T}}$, is measured through a pyranometer whose relative uncertainty is about $5 \%$. This uncertainty is assumed for both solar irradiance and solar energy $E_{G_{\mathrm{T}}}=\Delta t \Sigma_{i} G_{\mathrm{T}, i}$.

\subsection{Monthly performances}

The output thermal energy of the solar field (upstream of $\mathrm{HXs}$ ), of the secondary circuit to the tank (downstream of $\mathrm{HXs}$ ), and of the process circuits (to hot water and feed water) was calculated for each month of 2014. The results (normalized with $A_{\mathrm{a}}$ ) are reported in Figure 2, where $E_{\text {coll }}$ is the field energy (upstream of HXs), $E_{\mathrm{HXh}}$ the energy for hot water, $E_{\mathrm{HXf}}$ the energy for feed water, and the utilization ratios are defined as $u_{\text {coll }}=E_{\text {coll }} / E_{G_{\mathrm{T}}}$ and $u_{\mathrm{sys}}=\left(E_{\mathrm{HXh}}+E_{\mathrm{HXf}}\right) / E_{G_{\mathrm{T}}}$. Utilization ratios range from values of the order of $35 \%$ during summer months to values of the order of $10 \%$ during winter months. The heat exchanger HXs between the field and the tank exhibits losses of the order of $3 \%$, with little seasonal dependency. Conversely, tank losses depend on the season, being of the order of $5-10 \%$ in summer and $10-15 \%$ in winter. Thermal losses due to pipes, according to a theoretical estimate, are negligible. On average, the global integration efficiency is about $93 \%$.

The yearly totals of the quantities shown in Figure 2 are $E_{G_{\mathrm{T}}}=1310 \mathrm{MWh}, E_{\text {coll }}=415 \mathrm{MWh}, E_{\mathrm{HXh}}=323 \mathrm{MWh}$, $E_{\mathrm{HXf}}=62.7 \mathrm{MWh}$. Electric consumptions of hydraulic pumps have also been estimated from nominal power and operation hours, resulting to be of the order of $1 \%$ of the useful energy.

\subsection{Efficiency analysis}

In order to better assess the collector performances, the bin method was applied to analyse the field efficiency, defined as $\eta=P_{\mathrm{th}} /\left(G_{\mathrm{T}} A_{\mathrm{a}}\right)$. This was compared with the nominal data provided by the collector supplier, obtained from EN 12975 tests. As usual, the efficiency is expressed in terms of the reduced temperature $T^{*}=\left(<T>-T_{\mathrm{amb}}\right) / G_{\mathrm{T}}$, where $<T>$ is the average temperature inside the collectors and $T_{\mathrm{amb}}$ is the ambient temperature. The nominal stationary efficiency curve of the collectors is $\eta_{\mathrm{st}}=0.811-2.710 T^{*}-0.010 T^{* 2} G_{\mathrm{T}}$ (quantities measured in SI units). Using the thermal capacity and the incident angle modifiers provided by the tests, we derived the following quasi-dynamic model for the efficiency: $\eta_{\mathrm{qd}}=0.813\left[K_{\mathrm{b}}(\theta) G_{\mathrm{b}}+0.983 G_{\mathrm{d}}\right] / G_{\mathrm{T}}-2.710 T^{*}-0.010$ $T^{2} G_{\mathrm{T}}-\left(7050 / G_{\mathrm{T}}\right)(\mathrm{d}<T>/ \mathrm{dt})$. Here, $\theta$ is the incidence angle on the collectors, the subscripts "b" and "d" hold for the beam and diffuse components of the solar irradiance, and $K_{\mathrm{b}}(\theta)=1-0.08997(1 / \cos \theta-1)$ is the incident angle modifier for the beam component. In order to apply this model, we used the experimental data for $T^{*}, G_{\mathrm{T}}$, and $\mathrm{d}<T>/ \mathrm{dt}$, while we calculated the incidence angle $\theta$ theoretically from the equations of solar coordinates and we assumed $G_{\mathrm{d}} / G_{\mathrm{T}}=20 \%$.

The comparison between experimental data (restricted to points where the circuit pump operates at $100 \%$ and averaged on $10 \mathrm{~min}$ ) and quasi-dynamic model is reported in Figure 3a, for July 2014. The bin method was applied grouping data within intervals (bins) for $T^{*}$ with a width of 0.01 . At typical operating conditions, the field performs 20-25\% worse than expected from the quasi-dynamic model. On the other hand, the agreement with the model is much better at higher values of $T^{*}$. An effect not considered here is the flow rate difference between the field conditions and the test conditions, which could account for a few percent difference in the efficiency. Note also that the uncertainty of the single experimental efficiency data, propagated from thermal power and solar irradiance, is of the order of $6 \%$.

In Figure 3b, yearly data are reported. In the three bins with $T^{*}=0.04-0.07$, where most operation points lie, the bin efficiency is close to $50 \%$. The values of the average efficiencies in the different bins are reported in Table 1 , together with the standard deviations corresponding to the scattering within each bin.

\section{Conclusions}

In this paper, we have presented the performance analysis of a flat plate collector solar field located in Austria. The observed efficiencies appear to be $15-25 \%$ below the ones 

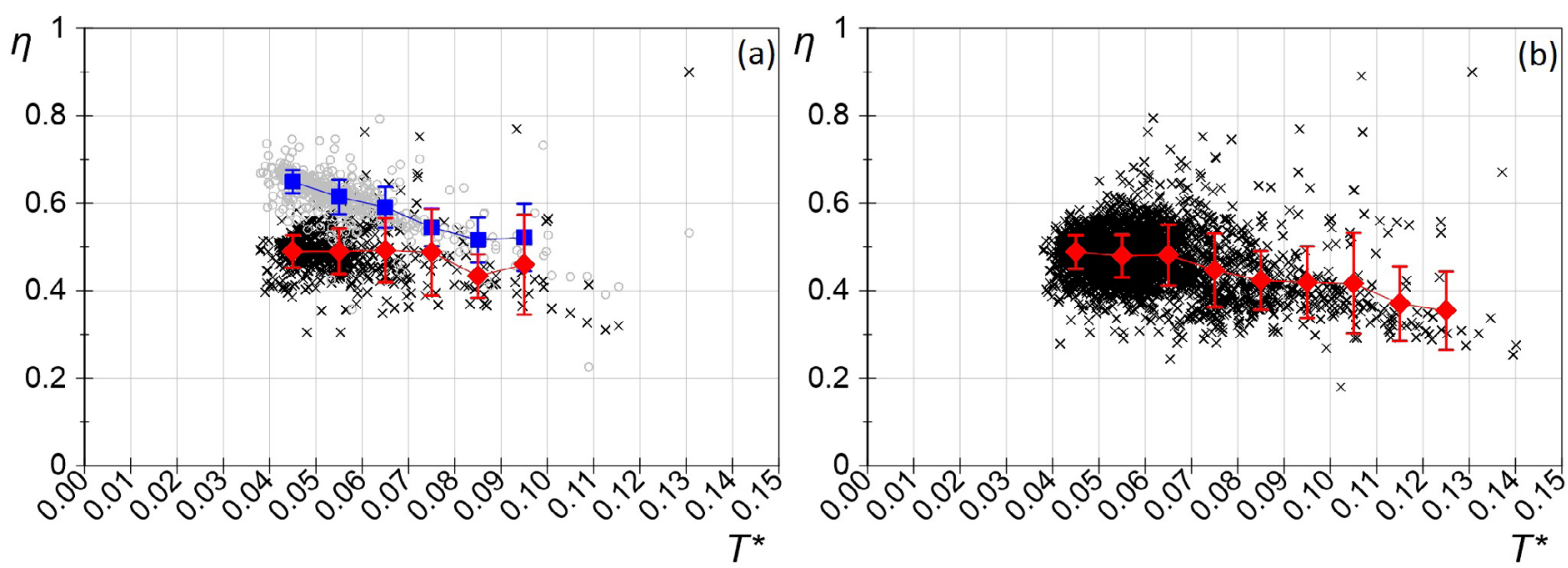

Fig. 3. Solar field efficiency as a function of the reduced temperature. Left panel (a): July 2014. Right panel (b): entire 2014. Grey circles: quasi-dynamic model calculated for the experimental operating conditions. Blue squares: bin averages of quasi-dynamic data. Black crosses: experimental data. Red diamonds: bin averages of experimental data. Bars: standard deviation within each bin.

Table 1. Bin efficiency and corresponding standard deviation referring to Figure 3(b).

\begin{tabular}{|c|c|c|c|c|c|c|c|c|c|}
\hline $\operatorname{Bin}\left(T^{*}\right)$ & $0.04-0.05$ & $0.05-0.06$ & $0.06-0.07$ & $0.07-0.08$ & $0.08-0.09$ & $0.09-0.10$ & $0.10-0.11$ & $0.11-0.12$ & $0.12-0.13$ \\
\hline $\begin{array}{l}\text { Bin } \\
\text { efficiency }(\eta)\end{array}$ & 0.489 & 0.479 & 0.481 & 0.447 & 0.424 & 0.420 & 0.417 & 0.371 & 0.355 \\
\hline $\begin{array}{l}\text { Standard } \\
\text { deviation }\end{array}$ & \pm 0.04 & \pm 0.05 & \pm 0.07 & \pm 0.08 & \pm 0.07 & \pm 0.08 & \pm 0.12 & \pm 0.09 & \pm 0.09 \\
\hline
\end{tabular}

expected from the quasi-dynamic model built with test data. This discrepancy can partly be due to flow rates different from test conditions; other effects can be related to conditions (dust, wind, radiative losses, actual diffuse fraction, etc.) not included in the model. The identification of a quasi-dynamic model fitting better to the field measurements could be the subject of future work. On the other hand, a very reliable operation was obtained during an entire year, showing that the efficiency in typical operating conditions is close to $50 \%$ and that the utilization ratio is stably of the order of $35 \%$ from March to September.

The research leading to these results has received funding from the European Union's Seventh Framework Programme FP7/20072013 under grant agreement No. ENER/FP7/296009/InSun.

\section{References}

1. S. Kalogirou, The potential of solar industrial process heat applications, Appl. Energy 76, 337 (2003)

2. C. Lauterbach, B. Schmitt, U. Jordan, K. Vajen, The potential of solar heat for industrial processes in Germany, Renew. Sustain Energy Rev. 16, 5121 (2012)

3. Ecoheatcool (IEE ALTENER Project), The European Heat Market, Work Package 1, Final Report Euroheat \& Power, 2006
4. I. Ben Hassine, M. Cotrado, R. Söll, D. Pietruschka, B. Gerardts, Operational improvements of a large-scale solar thermal plant used for heat supply in the ham production, Gleisdorf Solar, 154 (2014)

5. EN 12975:2006, Thermal Solar Systems and Components Solar Collectors - Part 1: general requirements, Part 2: test methods

6. EN ISO 9806:2013, Solar energy - Solar thermal collectors Test methods

7. B. Perers, P. Kovacs, U. Pettersson, Experiences and lessons learned from 30 years of dynamic collector testing, modelling and simulation, in Proceedings of the ISES Solar World Congress 2011 (2011)

8. M.C. Rodríguez-Hidalgo, P.A. Rodríguez-Aumente, A. Lecuona, G.L. Gutiérrez-Urueta, R. Ventas, Flat plate thermal solar collector efficiency: transient behavior under working conditions. Part I: model description and experimental validation, Appl. Therm. Eng. 31, 2394 (2011)

9. M.C. Rodríguez-Hidalgo, P.A. Rodríguez-Aumente, A. Lecuona, G.L. Gutiérrez-Urueta, R. Ventas, Flat plate thermal solar collector efficiency: transient behavior under working conditions. Part II: model application and design contributions, Appl. Therm. Eng. 31, 2385 (2011)

10. ISO/IEC Guide 98-3:2008, Uncertainty of measurement Part 3: guide to the expression of uncertainty in measurement

Cite this article as: Marco Cozzini, Roberto Fedrizzi, Mauro Pipiciello, Robert Söll, Ilyes Ben Hassine, Dirk Pietruschka, Monitoring of a flat plate solar thermal field supplying process heat, Renew. Energy Environ. Sustain. 1, 4 (2016) 